\section{Wenig sensitiver Schnelltest}

Mit FastCheckPOC ist seit Ende 2004 ein neuer Allergieschnelltest für $2 \times 12$ Allergene auf dem Markt. Geworben wird damit, dass bereits zwei Tropfen Blut zur Durchführung des Tests ausreichen. Das Blut wird in eine Testlösung pipettiert. Diese wird dann auf einen mit Allergenen und Kontrollen belegten Testfilter aufgetragen. Nach 15 Minuten wird die Blutlösung abgewaschen, gebundenes IgE mit einem Antikörper markiert und semiquantitativ nachgewiesen. Die gesamte Testdauer beträgt 30 Minuten. Positive Reaktionen, d.h. das Vorliegen einer allergischen Reaktion, erscheinen auf dem Testfilter als Pluszeichen (+), negative als Minuszeichen (-).

Es handelt sich bei dem Test um einen Enzym-Immunoassay mit geringer Sensitivität, wie bei der herstellenden Firma zu erfragen war. Der positive Nachweis fängt im Vergleich zum CAP-FEIA-System am Ende der Klasse 2 bzw. am Anfang der Klasse 3 an, also ab 2-3,5 kU/l. Dies gilt für Standardallergene wie Birkenpollenoder Hausstaubmilbenallergene. Für „schwierige“ Allergene wie z.B. Nahrungsmittelallergene existieren keine gesicherten Aussagen. Die niedrige Sensitivität bedeutet, dass viele falschnegative Ergebnisse produziert und somit schwächere Sensibilisierungen übersehen werden. Der Test lässt nur eine qualitative Ja-Nein-Aussage zu. Die Differenzierung zwischen Ja und Nein kann durch die nicht eindeutige Ablesbarkeit problematisch sein. Ein Messgerät zur Ergebnisauswertung existiert nicht. Studien zu den beschriebenen guten Korrelationen mit dem CAP-FEIASystem sind im Internetportal der Firma nicht dokumentiert. Detaillierte Studien bezüglich der Sensitivität und Spezifität des Nachweises der einzelnen Allergene bei Patienten mit verschiedenen Sensibilisierungsgraden sind zu fordern.

Eine Testeinheit kostet ca. $12 €$ plus Mehrwertsteuer - ob sich da eine Abrechnung im Oll-Kapitel des neuen EBM plus lohnt, bleibt dahingestellt. Von einem „Ouantensprung", wie publiziert wurde, kann bei diesem Test aber auf gar keinen Fall die Rede sein. Schließlich lautet bei der allergologischen Diagnostik die übliche Reihenfolge Anamnese - Hauttestung Serologie-Provokationstestung; insbesondere bei beabsichtigten Hyposensibilisierungen sollte diese Reihenfolge auch eingehalten werden.

Dr. Werner Kersten, Moers, für die In-vitro-Arbeitsgruppe des ÄDA
_ die notwendigen Anforderungen an die Notfallausstattung allergologisch tätiger Ärzte,

_ den grundsätzlichen Ablauf einer rationalen allergologischen Stufendiagnostik,

_ die Grundsätze der spezifischen Immuntherapie allergischer Erkrankungen insbesondere unter dem Aspekt der Risikovermeidung für den Patienten sowie

— Vorschläge zur Sicherung der Ergebnisqualität in der Allergologie.

\section{Qualitätsprofil Allergologe}

Zur persönlichen Qualifikation allergologisch tätiger Ärzte wird in diesen Empfehlungen ausgeführt:

- Allergologische Leistungen sind nach Facharztstandard - entsprechend den Vorgaben der jeweiligen gültigen (Muster-)Weiterbildungsordnung sowie der gültigen (Muster-)Richtlinien über den Inhalt der Weiterbildung der Bundesärztekammer - zu erbringen.

— Allergologische Leistungen mit hohem Gesundheitsrisiko für den Patienten sind durch Ärzte zu erbringen, die über spezielle Kenntnisse und Erfahrung im Umgang mit diesen Maßnahmen verfügen.

—Allergologische Leistungen können

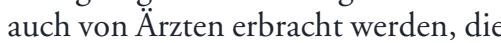
nach den Vorgaben früherer Weiterbildungsordnungen weitergebildet wurden, sofern eine auf die aktuell gültige Weiterbildungsordnung bezogene Qualifikation (z. B. durch Fortbildung, durch Nachweis der entsprechenden Berufserfahrung oder ggf. durch ein Kolloquium) nachgewiesen wurde.

— Der allergologisch tätige Arzt hat sich auf dem Gebiet der Allergologie und der Notfallmedizin fortzubilden. Er muss in der Lage sein, entsprechend den berufsrechtlichen Regelungen seine Fortbildungsaktivitäten gegenüber der Ärztekammer nachzuweisen. Empfohlen wird die Teilnahme an kurrikulären Fortbildungsmaßnahmen der medizinischen wissenschaftlichen Fachgesellschaften und der ärztlichen Berufsstände sowie die Beteiligung an fachspezifischen Qualitätszirkeln.

\section{Barfußallergologie im neuen EBM}

Umso schwerer ist es hinzunehmen, dass im neuen EBM 2000 plus außerhalb des Kapitels „Allergologie“ eine hausärztliche allergologische Basisdiagostik ( $\mathrm{Nr}$. 03340) vorgesehen ist. Der obligate Leistungsinhalt umfasst die allergologische Anamnese und eine Pricktestung mit mindestens zehn Tests, einmal im Behandlungsfall.

Man kann schon jetzt vorhersehen, dass der Außendienst etlicher Firmen ausschwärmen wird, um an Praktiker umsonst Testkästen zu verteilen und sie dazu zu animieren nach dieser Diagnostik, die „risikolose“ sublinguale Immuntherapie durchzuführen. Dieses Szenario ist vorprogrammiert und wird die Qualität in der Allergologie nicht steigern und dem Ruf dieses Bereiches abträglich sein.

Deshalb wird es auch 2005 ein Hauptziel des ÄDA sein, dass der Richtlinienentwurf zur Qualitätssicherung in der Allergologie als gültige Richtlinie verabschiedet wird sowie aus den Vorschlägen Qualitätsindikatoren und -kriterien abgeleitet werden, um auf diese Weise den Einfluss der vorgeschlagenen Maßnahmen auf die Versorgungsqualität messen zu können. Entsprechende Gespräche werden unter Leitung des ÄDAVorstandsmitgliedes Dr. Josef Wenning mit der KBV geführt.

Hoffentlich findet die Qualität auch in unserem Bereich der Medizin bald Eingang. Der ÄDA wird weiter berichten.

Dr. Werner Kersten, Moers 